\title{
Development of Dutch children's comprehension of subject and object wh-questions
}

\author{
The role of topicality
}

\author{
Iris Strangmann, Anneke Slomp and Angeliek van Hout \\ University of Groningen
}

\begin{abstract}
While Dutch welke 'which'-questions are structurally ambiguous, number agreement cues can disambiguate them. Despite such agreement cues, children misinterpret object questions as subject questions (Metz et al. 2010, 2012; Schouwenaars et al. 2014). We investigated if adding another cue, specifically, topicality in a discourse context, helps the interpretation of which-questions in two groups of Dutch children $(5 ; 5, \mathrm{n}=15$ and 8;5, $\mathrm{n}=21)$. Using a referent-selection task, we manipulated number on the verb and postverbal NP to create unambiguous $w$ h-questions. Moreover, the questions were preceded by a discourse which established a topic, relating either to the $w h$-referent or the postverbal NP referent. Nevertheless, both 5- and 8-year-olds misinterpreted object questions as subject questions, ignoring the number and topicality cues to resolve the (local) ambiguity of which-questions. Our results confirm the effect of a subject-first bias in children's interpretation of wh-questions. We conclude that topicality, in combination with number agreement, is not strong enough to overrule this subject-first bias.
\end{abstract}

Keywords: First language acquisition, wh-questions, object questions, topicality, subject-first bias, Dutch

\section{Introduction}

Research on the acquisition of wh-questions goes back to the 1970s (Ervin-Tripp 1970; Stewart \& Sinclair 1975). Many studies find an asymmetry in the comprehension of wh-questions: Subject questions are acquired earlier than object questions, and moreover, object questions are incorrectly interpreted as subject questions. This asymmetry has been established for various languages, including English 
(Stewart \& Sinclair 1975; Avrutin 2000; Philip, Coopmans, van Atteveldt \& van der Meer 2001; Deevy \& Leonard 2004), Italian (de Vincenzi, Arduino, Ciccarelli \& Job 1999), Hebrew (Friedman, Belletti \& Rizzi 2009) and Dutch (van der Meer, van Atteveldt, Coopmans \& Philip 2001; Metz, van Hout \& van der Lely 2010, 2012; Schouwenaars, van Hout \& Hendriks 2014). There is variation as to when children become sensitive to the grammatical cues that mark object questions in their language, which are different ones across languages (Van der Lely et al. 2010).

Dutch wh-questions typically have just one interpretation. Yet they are structurally ambiguous between a subject and an object reading: One cannot determine subject/object relations on the basis of the word order $\mathrm{WH}-\mathrm{V}-\mathrm{NP}$; there are also no systematic case cues. The Dutch question in (1) has two possible interpretations: The wh-phrase welke piraat can be interpreted as subject or object.

(1) Welke piraat wast de indiaan?

which pirate washes the Indian?

a. Subject question: 'Which pirate is washing the Indian?'

b. Object question: 'Which pirate is the Indian washing?'

Ambiguity hardly ever arises, however, as wh-questions are almost always disambiguated in one way or another. Dutch has case on pronouns which determines the grammatical relations, (2), so ambiguity only occurs when subject and object are full NPs, (1). Moreover, non-reversible verbs such as eat allow just one interpretation given their lexical semantics - pirates can eat apples, but not vice versa, (3). Ambiguity only occurs when the verb is reversible, (1). Animacy gives an additional cue: Animate NPs are typically subject and inanimate NPs object, (3) (although not always, cf. the truck is pulling the cow out of the ditch). Furthermore, the verbal context can make clear who is doing what to whom; the discourse in (4) suggests who is doing the washing (agent and subject) and who is going to be washed (theme and object). Finally, number agreement is a subtle, but syntactically hard cue in those cases where the number of wh-phrase and post-verbal NP differs. Given number agreement between the plural verb and post-verbal, plural noun in (5), the Indians is subject of the washing and which pirate is object.

(2) a. Welke piraat wast hem? which pirate washes him 'Which pirate is washing him?'

b. Welke piraat wast hij? which pirate washes he 'Which pirate is he washing?'

(3) Welke appel eet de piraat? which apple eats the pirate? 
'Which apple does the pirate eat?'

(4) a. Context: "When the pirates arrive on land, they are really dirty. Luckily there are some Indians there to help them get clean again."

b. Welke piraat wast deze indiaan? which pirate washes this Indian? 'Which pirate is this Indian washing?'

(5) Welke piraat wassen de indianen? which pirate.SG wash.PL the indians.PL? 'Which pirate are the Indians washing?'

Two studies with Dutch children aged 5 to 9 investigated the effect of number agreement on the interpretation of subject and object which-questions using unambiguous questions such as (5) (Metz et al. 2010, 2012; Schouwenaars et al. 2014). Number agreement did not help with the interpretation of object whichquestions: Virtually all object questions were interpreted as subject questions. The conclusion of these studies is that there is a strong subject-first bias: Children take the first NP in a sentence (i.e. question) as subject, despite the ungrammaticality of this interpretation given the number cues.

A prominent explanation as to why object questions are harder than subject questions uses Rizzi's theory of Relativized Minimality (Friedmann, Belletti \& Rizzi 2009; Friedmann \& Novogrodsky 2011; Jakubowicz 2011; Guasti, Branchini \& Arosio 2012). This minimality explanation states that interpretation is harder when the terms that are connected in the dependency relation between wh-phrase and its trace are separated by an intervener which could potentially be involved in that relation; in the case of object questions, the subject. In representation (6a) there is no intervener between the $w h$-phrase and the trace in subject position, but in (6b) the subject intervenes between the wh-phrase and the trace in object position, and is a potential minimality intervener.

$\begin{array}{lll}\text { (6) a. Subject question: } & \text { Wh }-\mathrm{V} \mathrm{O}_{-} \\ \text {b. Object question: } & \text { Wh } \mathrm{S}_{-}\end{array}$

The minimality explanation for the subject-object asymmetry posits that children experience difficulties when the object $w h$-phrase and the subject NP intervening between filler and gap have the same set of features, because that constitutes a minimality violation. The interference effect is less severe or even absent when the object wh-phrase and the subject are sufficiently dissimilar. This has been established for noun type in Hebrew: When object wh-phrase and subject both involve lexical nouns (which pirate, the Indian), children have difficulties, but with a whpronoun (who) and a lexical NP subject (the pirate), children perform better on 
object questions, as there is less interference (Friedmann et al. 2009; Friedmann \& Novogrodsky 2011). Adani, van der Lely, Forgiarini and Guasti (2010) extend the minimality account to number, arguing that there should be less interference when the number feature on the potential intervener differs. They investigated the effect of number (and gender) in Italian children's interpretation of relative clauses (which have the same structural ambiguity as Dutch wh-questions). There was indeed an effect of mismatching features: Better performance on conditions in which number on the noun heading the NP mismatched with the subject NP in the relative clause (the lion-SG that the crocs-PL are touching is sitting-SG) than in conditions where number matched (the lion-SG that the cat-SG is touching is sitting-SG). This supports the minimality explanation for the feature of number.

The object questions used in the Dutch studies mentioned above (and also in the present one) employ dissimilarity of number to disambiguate the questions: The which-phrase is singular and the post-verbal NP is plural - which pirate (SG) and the Indians (PL) in (5). However, there was no effect of mismatching features: Number mismatch between which-phrase and subject did not help Dutch children (up to age nine) on which-questions. It seems that the subject is such a strong intervener that object questions are always interpreted as subject questions, despite feature dissimilarity of $w h$-phrase and potential intervener. The Dutch studies conclude that the minimality account as extended to number features does not account for children's difficulty with Dutch object questions, even though it explains well the effects of number match/mismatch in Italian relative clauses, as well as noun type match/mismatch in Hebrew wh-questions. There must be some additional difficulty with object which-questions in Dutch.

Metz et al. $(2010,2012)$ suggest processing difficulties as an alternative explanation, referring to the length of the distance between filler and gap. The subjectobject asymmetry for wh-questions and also relative clauses is well known in the adult processing literature: Adults parse subject questions faster than object questions. The idea is that the parser tries to close the gap between filler (wh-phrase) and gap position as soon as possible - which is at the subject position, triggering the so-called 'Active-Filler effect' (Stowe 1986; Frazier \& Flores d'Arcais 1989). Deevy \& Leonard (2004) extend this processing explanation to acquisition. They argue that the distance between filler and gap matters; a longer distance requires more working memory in processing before the filler can be linked to its gap. Subject questions involve a shorter distance, hence they are easier to process than object questions. Metz et al. argue that children's problems with unambiguous object questions arise as a (kinder)gardenpath effect (Trueswell, Sekerina, Hill \& Logrip 1999): The child's parser takes the first NP to be subject. When the sentence later on shows incorrect number agreement between the alleged subject and the verb, the child's parser is not yet capable of revising its original, incorrect parse. 
Schouwenaars et al. (2014) propose an explanation framed in an Optimality Theory approach that involves misranked constraints. They advance three constraints that guide the interpretation of wh-questions: AGREEMENT guarantees subject-verb agreement; Wh-FIRST requires the $w h$-phrase to be the first element of the clause; and Subject-First says that the subject must come first. The adult ranking is shown in (7). Schouwenaars and colleagues argue that, in the child's grammar, AGREEMENT ranks below the other two constraints, (8): The subject and wh-phrase must come first, possibly at the expense of violating Agreement. With this ranking, children interpret any type of wh-question as a subject question, which accounts for their reversal errors with object which-questions.

(7) Agreement $>>$ Wh-First $>>$ SubjeCt-First

(8) Subject-First $>>$ Wh-First $>>$ AgreEment

Summarizing so far, across languages there is an asymmetry between the interpretation of subject and object $w h$-questions revealing a subject-first bias: Children take the first NP in the sentence (i.e. the $w h$-phrase in a question) as subject. Two studies on Dutch found that number agreement does not offer a strong enough cue to eliminate the subject first-bias in children. We have reviewed three types of accounts. The minimality explanation is a syntactic explanation and poses that the interpretation of a wh-object question is complicated by the intervening subject NP between the which-phrase and its gap. Feature dissimilarity can alleviate this interference effect. However, number mismatch in Dutch wh-questions apparently is not strong enough to overcome the minimality interference. The second explanation refers to processing: Not the properties of the intervening NP, but the greater distance between filler and gap would explain why adults process object $w h$-questions slower than subject $w h$-questions. With children it even leads to a (kinder)gardenpath effect: The child's parser gets stuck and is unable to revise its first choice, leading to a subject interpretation. The third explanation derives from Optimality Theory and employs the idea that children have not acquired the correct hierarchy of constraints: Agreement is not ranked high enough.

Are there any other cues that can help Dutch children to give an object-question interpretation for unambiguous which-questions (as disambiguated by number)? Potential cues discussed above include pronoun case, animacy and context. The present study is part of a larger research program that looks at these cues one by one. Here we investigate the role of context in combination with number agreement, specifically, topicality.

A proper discourse context can disambiguate between a subject or object interpretation in wh-questions, as illustrated in (4). The 'aboutness topic' is determined by the preceding discourse (Lambrecht 1994). Topics are often subjects 
(Givón 1983) and subjects are often agents. The information structure of the preceding discourse can help determine the subject and object relations in a sentence: When the verbal context establishes a certain referent as the topic, it will tend to be the subject of the next sentence. So, when the wh-phrase is not topic, that should be a clue that it is not subject either, because the topic is subject; a non-topic whphrase will be object.

Sensitivity to topicality in child learners has been investigated in a number of different language domains. Spenader, Smits \& Hendriks (2009) varied between the introduction sentences to test Principle B effects. Children aged 4-6 made fewer mistakes in a discourse where only one referent was introduced and that referent was also the topic, than in a discourse with two referents. Hollebrandse (2004) investigated universal quantifier interpretation in extra-object situations in children aged 4-8 (for example, Are all Indians riding a horse? in a situation with 3 Indians, each riding a horse and one extra horse without an Indian; target answer: yes; children's spreading answer: no). Children were sensitive to topicality: There were much fewer spreading errors when the quantified phrase was the topic in the preceding discourse. This effect only occurred with quantified subjects, not objects, which suggests that children know the prototypical mapping between topics and subjects. Wubs, Hendriks, Hoeks \& Koster (2009) investigated topicality with pronoun anaphora in production and comprehension in children aged 4-6. In this study, however, the children did not show any sensitivity to the discourse context. Both in the topic-continuity and topic-switch context, they produced pronouns instead of using a full NP to mark the topic switch in the latter condition. For determining the reference of pronouns, they were not sensitive to topic continuity or switch either, and selected either one of two referents in the discourse at similar rates, independent of the context.

There are several studies in the adult processing literature that show an effect of context when comparing sentences with a canonical, subject-first order versus a non-canonical, object-first word order (we did not find any adult parsing studies on the role of discourse context in wh-questions). In neutral contexts, German sentences with a canonical SO order are parsed quicker and accepted more often than those with a non-canonical OS order. However, a proper discourse context that makes the object topic, facilitates the processing of the non-canonical OS order (Meng, Bader \& Bayer 1999). This suggests that the parser is sensitive to topicality. An ERP study established the same sensitivity and pinpointed the modulating effect of context at the position of the fronted object in the non-canonical OS order (Bornkessel, Schlesewsky \& Friederici 2003), right where the parser makes an initial decision about the grammatical role of that fronted NP.

Whereas adults are sensitive to context when processing grammatical relations, the picture of children's acquisition of topicality is unclear: There is 
sometimes sensitivity (Hollebrandse 2004; Spenader, Smits \& Hendriks 2009), but also no sensitivity (Wubs, Hendriks, Hoeks \& Koster 2009). What about topicality and which-questions? We investigate if topicality - in conjunction with number - helps determine subject and object relations in which-questions. Is the combination of number plus topicality strong enough to overcome the subject-first bias? Since topics typically map onto subjects and subjects onto agents, we hypothesize that sensitivity to topicality helps the interpretation of object which-questions, because it adds an extra cue in addition to number agreement. When the which-NP is not topic, this is a clue that it is not the subject, but rather the object. So we predict that Dutch children are better at interpreting object which-questions when the subject (in post-verbal position) is topic of the preceding discourse than when the object (in the which-phrase) is topic.

Section 2 presents the method of our study on topicality and which-questions with Dutch 5- and 8-year-olds. Section 3 presents the results, which are discussed in Section 4.

\section{Method}

\subsection{Pretest}

We pretested children's comprehension of number agreement in declarative sentences, before testing them on subject and object which-questions, in order to establish that they were able to process singular and plural marking on the verb, which is the morpho-syntactic cue in the wh-test. 64 children participated in this pretest: 415 -year-olds and 228 -year-olds. The children were recruited from three different elementary schools. Also, there was a control group of twenty adults. All participants had Dutch as their native language.

The pretest, including all the materials, was borrowed from Schouwenaars et al. (2014). Children's ability to differentiate between singular and plural verbs was tested by means of a Picture Selection Task. The eight items varied between
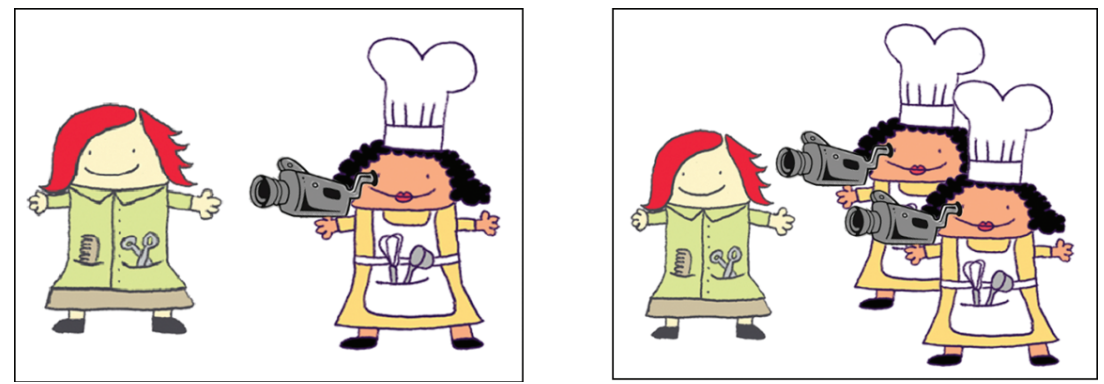

Figure 1. Sample item pretest, showing singular ze (left) and plural ze (right) 
singular and plural on the auxiliary. Two pictures were shown on a computer screen, Figure 1, while participants heard a prerecorded sentence, i.e. example (9).

(9) Ze heeft / hebben de kapster gefilmd.

ZE has /have the hair dresser filmed

'She has / They have filmed the hairdresser.'

In Dutch, the pronoun $z e$ is a feminine third person pronoun, underspecified for number: Singular pronoun 'she' or plural pronoun 'they (feminine)'. In (9) only number on the verb offers a cue to differentiate between a singular and plural $z e$. One picture matched the target sentence; the distracter showed an incorrect number of referents. Following Schouwenaars et al. (2014), the verbs in the prerecorded sentences were uttered in the present perfect, because in Dutch the difference between singular and plural is more salient on the auxiliary (heeft/hebben) than on simple present verb forms (filmt/filmen) (different vowels and consonants.)

The criterion to pass the pretest was set at $6 / 8$. If participants scored fewer than 6 correct, they did not continue. There was a large drop-out among the 5-year-olds: 26 out of 41 failed. This was not surprising seeing the drop-out rate in Schouwenaars et al., who tested 6- and 7-year-olds of whom about a third failed. Only participants who passed the pretest took the wh-questions test.

\subsection{Participants}

Table 1 shows an overview of the number of participants in all experimental groups.

Table 1. Number of participants and mean age in Pretest and Wh-questions test

\begin{tabular}{llll}
\hline & Pretest & Age range & Wh-test \\
\hline 5-year-olds & $\mathrm{n}=41(5 ; 5)$ & $4 ; 11-5 ; 11$ & $\mathrm{n}=15(5 ; 5)$ \\
8-year-olds & $\mathrm{n}=22(8 ; 5)$ & $8 ; 1-8 ; 11$ & $\mathrm{n}=21(8 ; 5)$ \\
Adults & $\mathrm{n}=20(25 ; 4)$ & $15 ; 0-37 ; 5$ & $\mathrm{n}=20(25 ; 4)$ \\
\hline
\end{tabular}

\subsection{Design and materials wh-questions}

The design of the which-questions test manipulated two independent variables: Topicality (Topic-first versus Topic-last) and Question Type (subject versus object) in a $2 \times 2$ design with four conditions. There were six items per condition, making 24 items in total. The sentences were disambiguated through different number on the verb (as shown in 10 and 11).

We used a Referent-Selection task using pictures with several referents, modeled after Hickock \& Avrutin (1996) and de Vincenzi, Arduino, Ciccarelli \& Job 
(1999). Figure 2 illustrates the sample subject and object questions in (10)-(11). The pictures were designed in such a way that they matched both interpretations: For a subject question, (10), the pirate on the right is the target referent; for an object question, (11), it is the pirate on the left. A singular auxiliary agrees with a singular subject, (10), indicating a singular agent; a plural auxiliary agrees with the plural subject in post-auxiliary position indicating a plural agent, and an object interpretation, (11).

(10) Welke piraat heeft de indianen gewassen? which pirate-SG has-SG the indians-PL washed? 'Which pirate washed the Indians?'

(11) Welke piraat hebben de indianen gewassen? which pirate-SG have-PL the indians-PL washed 'Which pirate did the Indians wash?'

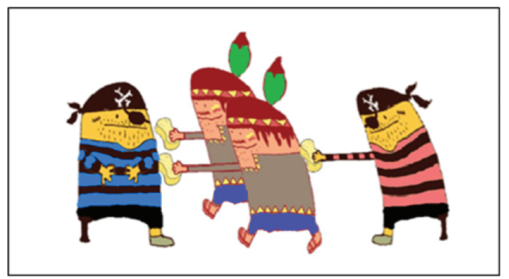

Figure 2. Sample picture, showing one pirate as agent and one as theme

Six reversible verbs were used: duwen (push), wassen (wash), kietelen (tickle), schoppen (kick), slaan (hit) and vangen (catch). The pictures showed eight different characters; every picture showed either all male or all female characters to prevent participants from imposing certain gender assumptions.

Before the participants heard the test question, a short story was told. The stories in (12) illustrate the two different ways of establishing topicality for the test sentences (10)-(11) and Figure 2.

(12) Stories creating topicality

a. Topic-First: "There are two pirates and two Indians here. The pirates have a black eye patches. They are wearing bandanas. And they're wearing black pants. Look, something is happening."

b. Topic-Last: "There are two Indians and two pirates here. The Indians have a green feather on their head. They've got brown hair. And they're wearing blue pants. Look, something is happening."

In the Topic-First condition, the first NP encountered in the first sentence (which pirate) is the topic of the story. In the Topic-Last condition, the last NP encountered in the sentence (the Indians) is topic. As discussed in 1.4, the topic is typically 
the subject of the sentence. This offers a cue for determining the grammatical relations: If topic becomes subject, the subject will be the first NP (i.e. wh-phrase) in the Topic-First condition, and the final NP in the Topic-Last condition. In the latter case, the $w h$-phrase is then the object. If children are sensitive to topicality and its potential effect on grammatical relations, we expect children to do better on object questions in the Topic-Last condition. In all test conditions, the whichquestions are disambiguated through the number agreement cue on the verb.

\subsection{Procedure}

Participants first took the pretest, and if they passed, they immediately continued with the wh-questions test. All participants were tested in a quiet room in school. The tests were presented via a PowerPoint presentation; the stories and test questions were prerecorded. The participants were instructed that they would hear a story. At the end of the story, there would be a question, which they could answer by pointing at a referent. One experimenter explained the task; the other noted down the answers. There was no time pressure. If children waited a long time before pointing, the experimenter asked them if they wanted to hear the story again. This was rarely necessary though.

\section{Results}

The results show clearly that children had significantly more difficulties when interpreting object questions compared to subject questions, in contrast to the adults. Table 2 shows the average percentages of correct responses (\%) and the corresponding standard deviations (SD) per condition for both groups of children and the adult control group.

Table 2. Average percentages of correct responses and standard deviations

\begin{tabular}{|c|c|c|c|c|c|c|}
\hline & \multicolumn{2}{|c|}{ 5-year-olds } & \multicolumn{2}{|c|}{ 8-year-olds } & \multicolumn{2}{|l|}{ Adults } \\
\hline & $\%$ & SD & $\%$ & $\mathrm{SD}$ & $\%$ & SD \\
\hline TOPIC-FIRST SUBJ-Wh & 100 & 0 & 99.24 & 3.55 & 100 & 0 \\
\hline TOPIC-LAST SUBJ-Wh & 100 & 0 & 96.96 & 6.58 & 99.17 & 3.72 \\
\hline TOPIC-FIRST OBJ-Wh & 3.7 & 7.35 & 8.33 & 19.07 & 98.33 & 5.13 \\
\hline TOPIC-LAST OBJ-Wh & 3.7 & 7.35 & 6.81 & 11.1 & 96.67 & 8.72 \\
\hline
\end{tabular}

A Kolmogorov-Smirnov test showed that the greater part of the children's data was not distributed normally, so we carried out non-parametric tests and used paired t-tests for the normally distributed data. Due to the high number of comparisons, 
we used a Bonferonni correction, dividing the level of significance by the number of statistical tests (twelve). The new level of significance was set at 0.004 .

A Mann-Whitney test showed that there is a significant effect of sentence type. Topic-First subject questions are interpreted correctly significantly more often than Topic-First object questions. This effect occurs in both the 5-year-olds and 8 -year-olds (both at $p<0.001$ ). T-tests showed that this also holds for Topic-Last subject and object questions. (5-year-olds: $\mathrm{t} 2(15)=28.49, p<0.001,8$-year-olds: $\mathrm{t} 2(21)=38.01, p<0.001)$. Further $\mathrm{t}$-tests showed that there were no significant effects of Topicality in any age group.

\section{Discussion}

There is a well-established asymmetry in the acquisition of wh-questions: Children initially interpret object $w h$-questions as subject $w h$-questions. Hebrew children do well on object questions around age 5 (Friedmann et al. 2009), but the problem persists in Italian and Dutch learners up to age 8-9 (de Vincenzi et al. 1999; Metz et al. 2010; Schouwenaars et al. 2014). Two Dutch studies concluded that number agreement as a single, morpho-syntactic cue is too weak: Children do not pick it up to correctly interpret object questions. This raises the question what can help children understand object questions? Candidate cues are pronoun case, animacy and context. The present study focuses on context, investigating if topicality, in conjunction with number agreement, helps understanding object which-questions. The test questions were syntactically disambiguated by number agreement and they were embedded in a short discourse that marked one set of referents as topic. If children are sensitive to the mapping between topicality and grammatical relations, they would be expected to interpret object which-questions correctly when it was not topic of the preceding discourse, because topics tend to be subjects and non-topics tend to be objects. Our findings replicate those of the two previous Dutch studies: Children up to age 8 effectively ignored the number cue, as they gave a subject interpretation to object which-questions, which was ungrammatical given the number on $w h$-phrase, verb and post-verbal subject. Thus, the combination of topicality and number was not sufficient in this study either: 5- and 8-yearolds interpreted object questions as subject questions. We conclude that the two cues together - number and topicality - were insufficient to overcome children's subject-first bias.

Dutch children's persistent ignoring of number in which-questions presents a more detailed picture of Friedman et al's (2009) minimality explanation. This states that interpreting an object question is difficult when the subject, which intervenes between filler and gap, has the same feature set as the wh-phrase, but becomes 
easier when they are sufficiently different. The question that rises in this account is as follows: Just how different do $w h$-phrase and potential intervener have to be for the intervention effect to disappear? Friedmann and colleagues found that it diminishes in Hebrew object questions when wh-phrase and intervener had mismatching noun type features (pronoun vs. lexical noun phrase). Adani, van der Lely, Forgiarini \& Guasti (2010) found that it also diminishes in Italian relative clauses when the relative clause head and intervener had mismatching number (singular vs. plural). In our study the object $w h$-phrase and the subject both contained lexical nouns, but differed in number: The $w h$-phrase was singular and the subject was plural. Still, the children did not interpret object questions correctly. So, for Dutch, in contrast to Italian, number mismatch does not constitute a large enough difference between $w$ h-phrase and intervener.

Why would number in Italian help diminish the intervention effect, whereas it does not have any effect in Dutch? A straightforward explanation is that the developing parser in Italian children is more attuned to number because verbal number inflection is crucial in a pro-drop language. Number on the verb establishes the reference of the subject in sentences without an overt subject. Number is therefore an important parsing cue in Italian. This sensitivity to number also applies to dependency relations in relative clauses. In Dutch, on the other hand, number on the verb is a mere morpho-syntactic reflex of subject-verb agreement; reference of the subject NP relies fully on the subject NP itself, not the verb. So the developing parser in Dutch does not need to pay crucial attention to number on the verb. The idea that the Dutch parser is not robustly attuned to number agreement is in fact supported by our own findings in the pretest: Out of 415 -year-olds only 15 children robustly employed number on the verb to determine reference of the ambiguous pronoun $z e$ ('she', they-feminine). Our finding that number does not help with the intervention effect of which-questions is therefore consistent with the processing explanation advanced by Metz et al. $(2010,2012)$, who argue that a kindergardenpath effect occurs when a child parses object $w h$-questions: The child parser takes the first NP it encounters (i.e. the $w h$-phrase) to be subject. The parser is unable to revise its initial choice later on in the sentence when it encounters a number agreement mismatch. Topicality does not interfere with this parsing strategy; topicality does not influence this first parser choice.

Certain acquisition studies established sensitivity to topicality (Hollebrandse 2004; Spenader et al. 2009). Our study shows that topicality does not help to establish the grammatical relations in object questions. Why not? Hollebrandse (2004) found an effect of topicality in his study on the interpretation of universal quantifiers, but only when the quantified phrase was subject; when it was object, the children did not improve. Hollebrandse concluded that there is a direct mapping from an Information Structure module with topic structure to the syntactic module. 
Taking his conclusions one step further and integrating them with our present results, we argue that the natural alignment between information structure and syntax depends on three interacting elements: Information structure (topicality), grammatical relations (subject) and word order (first position). When all three are perfectly aligned - the topic is subject in first position - interpretation is straightforward. In the case of subject questions, this is indeed the case. However, in the case of object questions, the alignment is off balance: The object wh-phrase is in first position; the subject is not. Lack of alignment between information and syntactic structure explains why there were no effects of topicality.

Another explanation for the lack of a topicality effect could be that we did not sufficiently establish topicality in our test. While the discourse context established the aboutness topic, the test sentences used a lexical NP to refer to it. Prototypical topics, however, are pronominal, certainly in a discourse that has clearly marked the referent as topic. Hence, the topicality cue that we provided here may not have been clear (or strong) enough. The topicality sensitivity in Spenader et al.s study (2009) was with pronouns: The discourse introduced a topic which was referred to with a pronoun in the test sentence (Here is a crocodile. The elephant is hitting him). A next step in our research program would be to examine object questions where the $w h$-phrase is a lexical NP and the subject is pronominal (Welke piraat hebben zij gewassen? 'which pirate did they wash?'). Note that such questions offer more cues, in addition to topicality: There is also subject case on the pronoun, and moreover, there is a NP type mismatch (pronoun vs. lexical noun).

Possibly, children do not rely much on grammatical cues at all when they interpret $w h$-questions. Rather, in a given situation it is often clear who is doing what to whom, and so this information determines the grammatical relations, see (4) above. Ongoing work in our lab investigates the effect of a who-is-doing-what-towhom context on the interpretation of object questions. Preliminary results show that children are not sensitive to this type of context either. They still interpret object questions (unambiguous ones as determined by number) as subject questions, despite a discourse set-up that spells out who is doing the action and to whom it is done (Strangmann \& van Hout in progress). This suggests that the subject-first bias is very strong and hard to overrule.

Finally, a very different explanation for the lack of topicality effects is that our task involves a meta-linguistic awareness rather than grammar or parsing. Adults may have performed so well on the task because they received grammar training in school, while children lack such metalinguistic skills. One interesting possibility would be to test illiterate Dutch adults on object which-questions. Such an experimental group could help distinguish between a linguistic explanation versus a metalinguistic explanation. ${ }^{1}$ 


\section{Conclusions}

This study presents another chapter in a series of studies on the acquisition of Dutch wh-questions. Our focus was the effect of topicality in conjunction with number. Dutch 5- and 8-year-olds interpreted object questions as subject questions, no matter whether the topic matched the subject or the object. We conclude that neither number nor topicality helps with the interpretation of object wh-questions; the subject-first bias is too strong. Future research should investigate the effect of other grammatical cues such as pronoun case, animacy and context in order to find out how children can overcome this bias.

\section{Notes}

* We thank the pupils and teachers of the OBS Het Rastholt, Hoogeveen, CBS De Tol, Zuidlaren en KBS Sint Clemens, Steenwijk for their cooperation. We thank the audiences at the University of Groningen Acquisition Lab and the TiN dag 2014 for their discussion and feedback. We also thank Petra Hendriks for letting us use pictures from her VICI-project 'Asymmetries in Language' and Atty Schouwenaars for sharing her pretest materials with us.

1. We thank an anonymous reviewer for this suggestion.

\section{References}

Adani, Flavia, Heather K.J. van der Lely, Matteo Forgiarini \& Maria Teresa Guasti. 2010. Grammatical feature dissimilarities make relative clauses easier: A comprehension study with Italian children. Lingua 120. 2148-2166.

Avrutin, Sergey. 2000. Comprehension of Wh-questions by children and Broca's aphasics. In Yosef Grodzinsky, Lewis P. Shapiro \& Dan A. Swinney (eds.), Language and the Brain: Representation and Processing, 295-312. San Diego: Academic Press.

Bornkessel, Ina, Matthias Schlesewsky \& Angela Friederici. 2003. Contextual information modulates initial processes of syntactic integration: The role of inter- versus intrasentential predictions. Journal of Experimental Psychology: Learning, Memory, and Cognition 29. 871-882.

Deevy, Patricia \& Lawrence B. Leonard. 2004. The comprehension of WH-questions in children with specific language impairment. Journal of Speech, Language and Hearing 47. 802-815.

Ervin-Tripp, Susan. 1970. Discourse agreement: How children answer questions. In John R. Hayes (ed.), Cognition and Development of Language, 76-106. New York: Wiley.

Frazier, Lyn \& Giovanni B. Flores d'Arcais. 1989. Filler driven parsing: A study of gap filling in Dutch. Journal of Memory and Language 28. 31-344.

Friedman, Naama, Adriana Belletti \& Luigi Rizzi. 2009. Relativized relatives: Types of intervention in the acquisition of A-bar dependencies. Lingua 119. 67-88.

Friedmann, Naama \& Rama Novogrodsky. 2011. Which questions are most difficult to understand? The comprehension of wh-questions in three subtypes of SLI. Lingua 121. 367-382. 
Givón, Talmy. 1983. Topic continuity in discourse: An introduction. In Talmy Givón (ed.), Topic Continuity in Discourse: A Quantitative Cross-language Study, 5-41. Amsterdam: John Benjamins.

Guasti, Teresa, Chiara Branchini \& Fabrizio Arosio. 2012. Interference in the production of Italian subject and object wh-questions. Applied Psycholinguistics 33. 185-223.

Hickok, Gregory \& Sergey Avrutin. 1996. Comprehension of wh-questions in two Broca's aphasics. Brain and Language 52. 314-327.

Hollebrandse, Bart. 2004. Topichood and quantification in L1 Dutch. International Review of Applied Linguistics Teaching 42. 203-2015.

Jakubowicz, Celia. 2011. Measuring derivational complexity: New evidence from typically developing and SLI learners of L1 French. Lingua 121. 339-351.

Lambrecht, Knud. 1994. Information structure and sentence form: Topic, focus and the mental representations of discourse referents. Cambridge: Cambridge University Press.

van der Lely, Heather \& COST Consortium. 2010. How do 5-year-olds understand questions: Differences in languages across Europe. Presented at Let the children speak: Learning of critical language skills across 25 Languages, Wellcome Trust Conference Center, London.

van der Meer, Matthijs, Wouter van Atteveldt, Peter Coopmans \& William Philip. 2001. Subject-object asymmetry in Dutch children's comprehension of wie-questions. In Ton van der Wouden \& Hans Broekhuis (eds.), Linguistics in the Netherlands 2001, 167-176. Amsterdam: John Benjamins.

Meng, Michael, Markus Bader \& Joseph Bayer. 1999. Die Verarbeitung von Subjekt-Objekt Ambiguitäten im Kontext [The processing of subject-object ambiguities in context]. In Ipke Wachsmuth \& Bernhard Jung (eds.), Proceedings der 4. Fachtagung der Gesellschaft für Kognitionswissenschaft, 244-249. St. Augustin: Infix Verlag.

Metz, Marijke, Angeliek van Hout \& Heather van der Lely. 2010. Dutch children's processing of wh-questions: Comprehension of who and which-questions in five- to nine-year-old children. Groninger Arbeiten zur Germanistischen Linguistik (GAGL) 51. 27-41. http://irs. ub.rug.nl/dbi/4ef4a1ef1c357

Metz, Marijke, Angeliek van Hout \& Heather van der Lely. 2012. Subject interpretation of object questions by Dutch 5-year-olds: The role of number agreement in comprehension. In Marion Elenbaas \& Suzanne Aalberse (eds.), Linguistics in the Netherlands 2012, 97-110. Amsterdam: John Benjamins.

Philip, William, Peter Coopmans, Wouter van Atteveldt \& Matthijs van der Meer. 2001. Subjectobject asymmetry in child comprehension of Wh-questions. In Anna H.-J. Do, Laura Dominguez \& Aimee Johansen (eds.), Proceedings of the 25th BUCLD, 587-598. Somerville: Cascadilla Press.

Schouwenaars, Atty, Angeliek van Hout \& Petra Hendriks. 2014. Word order overrules number agreement: Dutch children's interpretation and production of which-questions. In ChiaYing Chu, Caitlin E. Coughlin, Beatriz Lopez Prego, Utako Minai \& Annie Tremblay (eds.), Selected proceedings of the 5th Conference on Generative Approaches to Language Acquisition North America (GALANA 2012), 60-71. Somerville: Cascadilla Proceedings Project.

Spenader, Jennifer, Erik-Jan Smits \& Petra Hendriks. 2009. Coherent discourse solves the pronoun interpretation problem. Journal of Child Language 36(1). 23-52.

Stewart, Jean \& Hermine Sinclair (1975). Comprehension of questions by children between 5 and 9. Linguistics 151. 17-26.

Stowe, Laurie. 1986. Parsing WH-constructions: Evidence for on-line gap location. Language and Cognition Processes 1. 227-245. 
Strangmann, Iris \& Angeliek van Hout. In progress. Context and the acquisition of Dutch object which-questions. Ms., University of Groningen.

Trueswell, John, Irina Sekerina, Nicole M. Hill \& Marian L. Logrip. 1999. The kindergartenpatheffect: Studying on-line sentence processing in young children. Cognition 73. 89-134.

de Vincenzi, Marica, Lisa S. Arduino, Laura Ciccarelli \& Remo Job. 1999. Parsing strategies in children comprehension of interrogative sentences. In Sebastiano Bagnara (ed.), Proceedings of the European Conference on Cognitive Science, 301-308. Rome: Instituto di Psicologia del CNR.

Wubs, Ellis, Petra Hendriks, John Hoeks \& Charlotte Koster. 2009. Tell me a story! Children's capacity for topic shift. In Jean Crawford, Koichi Otaki \& Masahiko Takahashi (eds.), Proceedings of the 3rd Conference on Generative Approaches to Language Acquisition North America (GALANA 3), 313-324. Somerville: Cascadilla Proceedings Project.

\section{Authors' addresses}

Iris Strangmann

University of Groningen

Nieuwe Blekerstraat 40A

9718 EK Groningen, The Netherlands

i.strangmann@rug.nl

Angeliek van Hout

Department of English

University of Groningen

Oude Kijk in 't Jatstraat 26

9712 EK Groningen, The Netherlands

a.m.h.van.hout@rug.nl

\author{
Anneke Slomp \\ University of Groningen \\ Verlengde Nieuwstraat 18A \\ 9724 HC Groningen, The Netherlands \\ a.slomp@student.rug.nl
}

